\title{
Influence of pulse magnetic fields treatment on optical properties of GaAs based films
}

\author{
R.V. Konakova, M.V. Sosnova, S.M. Red'ko, V.V. Milenin, R.A. Red'ko \\ V. Lashkaryov Institute of Semiconductor Physics, National Academy of Sciences of Ukraine, \\ 45, prospect Nauky, 03028 Kyiv, Ukraine \\ Phone: +38 (044) 525-94-64; fax:+38 (044) 525-61-82, e-mail: redko.rom@gmail.com
}

\begin{abstract}
Long-term transformations of the optical reflectance of GaAs epitaxial structure under weak magnetic field treatment $(B=60 \mathrm{mT}, f=10 \mathrm{~Hz}, \tau=1.2 \mathrm{~ms}, t=$ $5 \mathrm{~min}$ ) have been obtained. Optical measurements were performed within the wavelength range $800 \ldots 1100 \mathrm{~nm}$ at $300 \mathrm{~K}$. Non-monotonous changes of reflectance were observed. Experimental results have been interpreted in terms of diffusion of point defects, resulting from destruction of metastable complexes (probably $\left[\mathrm{V}_{\mathrm{As}}+\mathrm{impurity}\right]$ ), from the internal boundaries to the surfaces of the investigated structures. The method for detection of non-equilibrium complexes in multilayer objects has been proposed.
\end{abstract}

Keywords: optical density, weak magnetic field, impurity-defect composition.

Manuscript received 31.01.14; revised version received 09.04.14; accepted for publication 12.06.14; published online 30.06.14.

\section{Introduction}

Magnetic fields with magnitudes much smaller than $1 \mathrm{~T}$ are widely used for improving mechanical properties of solids [1] as well as for acceleration of chemical reactions in biologic objects [2]. For these values of magnetic induction, the relation $\mu_{\mathrm{B}} B<<k T$, with $\mu_{\mathrm{B}}$ being the Bohr magneton, can be accepted. Treatments under these magnetic fields occur with low energy absorption, considerably smaller than $k T$ value at room temperature. Therefore, traditional approach cannot be used to explain the observed changes of macroscopical characteristics of the materials under study. Alternative mechanisms were proposed in different reports [1-4], which, however, do not explain all experimental results. In particular, this refers to transformation of the low-frequency region in spectral dependences [5]. So, investigations in this direction are required to understand all the processes induced by weak magnetic fields (WMF).

\section{Experimental}

The homoepitaxial thin films (thickness $\sim 0.15 \mu \mathrm{m}$ ) of GaAs were prepared on substrate with the thickness $350 \mu \mathrm{m}$, using a buffer layer $(2 \mu \mathrm{m})$. The concentrations of carriers in layers were $6 \cdot 10^{16}, 2 \cdot 10^{18}$ and $6 \cdot 10^{15} \mathrm{~cm}^{-3}$, respectively. Measurements of optical reflection (OR) were carried out at room temperature at the angles of measuring beam incidence $15^{\circ}, 30^{\circ}$ and $45^{\circ}$ within the spectral range $800 \ldots 1100 \mathrm{~nm}$. We used two types of beam polarization, $s$ and $p$. The spectral dependences of the light reflectance for these epitaxial structures were measured using an automated setup based on IKS-12 spectrometer equipped with a device for measuring the angular dependence of reflectance. Since the treatment regime with parameters $B=60 \mathrm{mT}, f=10 \mathrm{~Hz}, \tau=1.2 \mathrm{~ms}$ was the most efficient for bulk III-V semiconductors [6], it was chosen for our experiments. The duration of processing varied within 5 min. 


\section{Results and discussion}

Spectral dependences of the optical reflectance at different angles of incidence for both polarizations are presented in Fig. 1. The initial state of the optical reflectance spectra for investigated structures was typical to gallium arsenide (Fig. 1, reference curves) with the characteristic inflexion around $900 \mathrm{~nm}$. In the long-wave region of the reference spectrum, the non-monotonous changes with the wavelength value were observed with small point dispersion. As it was established, appearance of interference in multilayered structures can occur due to the reflection of wave from two different interfaces. Using the expression for the maximum shift of phases $\sim 1$ :

$$
d=\frac{m \lambda_{m_{1}} \lambda_{m_{2}}}{2 \sqrt{\left(n^{2}-\sin ^{2} \alpha\right)\left(\lambda_{m_{2}}-\lambda_{m_{1}}\right)}},
$$

where $m$ is the number of oscillations between two extremes corresponding to $\lambda_{m_{1}}, \lambda_{m_{2}} ; n$ is the refractive index, it is possible to estimate the thickness $d$ of the epitaxial layer of the semiconductor structure that provides interference. The value $d=2.57 \mu \mathrm{m}$ was obtained for the initial state, which disagreed with the thickness of the top layer of the device structure, but agreed sufficiently with the sum of the thicknesses of active and buffer layers $(2.15 \mu \mathrm{m})$. We can conclude, therefore, that the interference takes place both in the double layer and the thin film with the optical thickness of $2.57 \mu \mathrm{m}$.

The spectral dependence of as-treated samples did not change. Four days later, the level of the lowfrequency shoulder $(>900 \mathrm{~nm})$ of the OR curve increased as compared to that of the reference one (Fig. 1, asterisks). The "distance" between the initial curve and the curve corresponding to the 4-day stored sample changed from 0.025 up to $0.060 \mathrm{arb}$. units of reflectance (by 25\%) for different incidence angles. The characteristics of oscillations (number of peaks and their frequency positions) also changed. Further OR measurements (Fig. 1, 7-day curve (triangles)) revealed continued modification of the spectral dependence in the long-wave region. The value of the optical thickness obtained using the expression(1) increased up to $3 . .4 \mu \mathrm{m}$. No oscillation processes were observed on the 25-th day after the WMF treatment. Only at $s$ polarization and at the angle $30^{\circ}$ some feeble oscillations were present. But after 60 days, we observed the return to the initial position with clear interference without point dispersion as well as the shift of the high energy parts of spectra to the blue region. This time period is in good agreement with 30- to 60-day period obtained from the photoluminescence measurements of bulk III-V materials [6].

Reliable reproducibility is observed for all the angles used in the experiments. The change of polarization from $s$ to $p$ does not change the main features of magneticinduced modifications (Figs 1a and 1b). Initially, after the
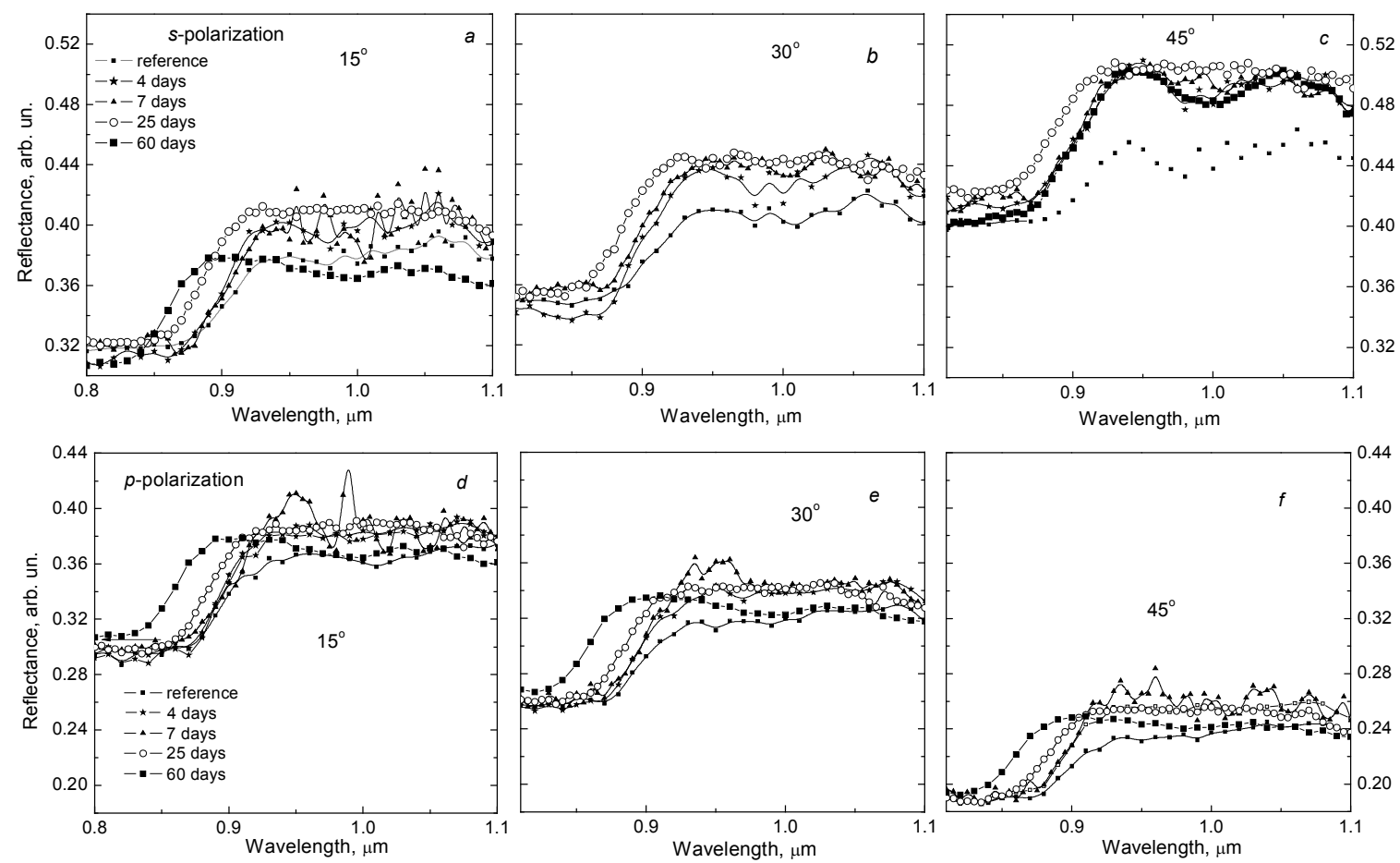

Fig. 1. Evolution of the spectrum of the optical reflectance inherent to epitaxial GaAs layers at different angles and polarizations $[(a)$ to $(c)$ - for $s$ and $(d)$ to $(f)$ - for $p$ ] after WMF treatment (small empty squares - initial state, asterisks - 4 days after treatment, triangles -7 days after treatment, circles -25 days after treatment, big filled squares -60 days after treatment). 
WMF treatment (4 days), the increase of optical reflectance is observed; then the increase of the thickness $d$ is observed. With increase of time, the OR spectra return to their initial states. Using all the calculated values, Fig. 2 was plotted. One can see that after the WMF treatment, the number of the observed peaks and the average value of $d$ initially increase. But after two months, the latter value becomes a little smaller than the initial one.

The observed modifications can be explained as follows. OR spectra can change due to two factors, namely, the change of the optical properties of the studied object ( $n$ or $k$ ) or the thickness of the layer forming interference. In the first case, more than $10 \%$ change is needed for the observed effects. We can, therefore, think that the optical constants do not change, but the second case is defining. The action of magnetic field can result in destruction of nonequilibrium complexes, existing at the interfaces between different layers of the studied objects [4]. Point defects (e.g., As vacancies) appear in this case. Then $\mathrm{V}_{\text {As }}$ migrates to the surface region. It causes degradation of clean boundaries between the layers in the bulk of the studied material and the increase of the estimated thickness $d$. Diffusion of arsenic vacancies to the main drain is analogous to the enrichment of surface by $\mathrm{Ga}$ atoms. It should result in the increase of reflectance. The latter point is confirmed by the experiment as well as by calculations. The simulated OR spectrum for the epitaxial GaAs obtained at $15^{\circ}$ is in good agreement with the thickness of epitaxial layers, the reflectance value, and the maxima of interference (Fig. 3). One can see that the increase of the thicknesses of top layers is accompanied by the increase of the number of interference peaks. This confirms a validity of our suggestions.

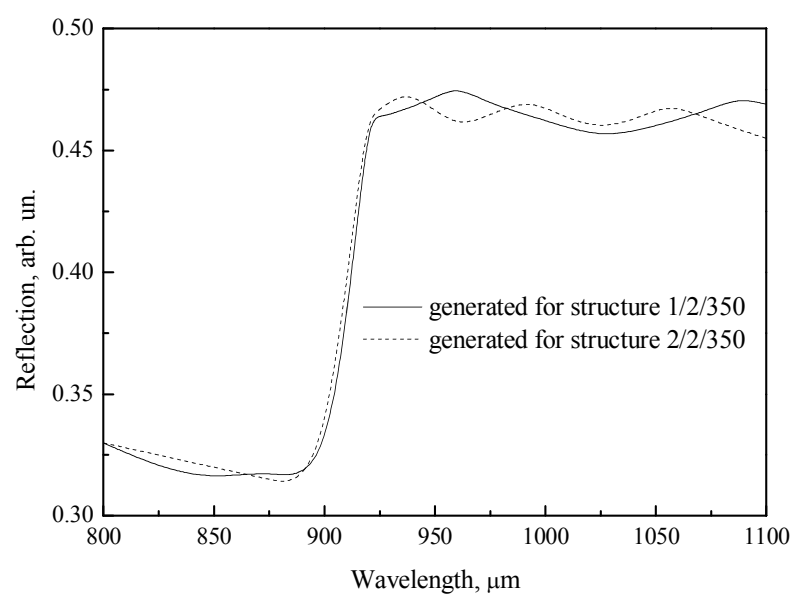

Fig. 2. Simulated curves of OR for multilayer structures top/buffer/substrate: 1/2/350 ( $\mu \mathrm{m})$ (solid) and 2/2/350 ( $\mu \mathrm{m})$ (dash).

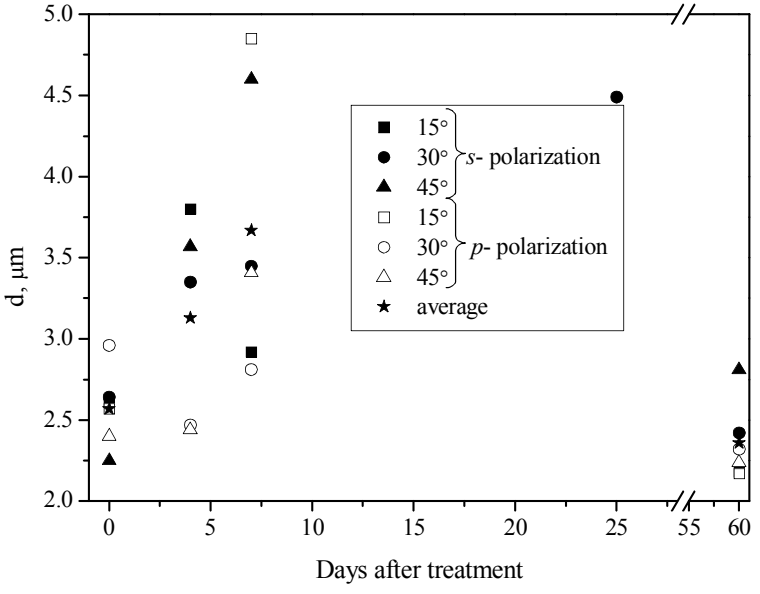

Fig. 3. Modification of the optical thickness $d$ in the course of experiments.

So, the physical interpretation of the observed modifications is based on the model proposed in [4]. As it was developed in [6], the surface of semiconductor due to WMF treatment is covered by metal component clusters that are mostly unstable and can be destroyed later. After 60 days, the state is similar to the initial one but with a smaller optical thickness $d$ and more prominent and clear interference processes in the lowfrequency shoulder of OR curves (Fig. 1, big filled squares). This fact testifies about the increase of the structural perfection of the internal boundaries of the investigated semiconductor material, probably due to the lower defect concentration.

The experimental data obtained in this work testify about two WMF-stimulated effects that were detected by the optical method, namely, the increase of reflectance and modification of internal boundaries between the layers of epitaxial structures. The action of magnetic field with small values of $B$ results in the long-term transformation of the defect structure of the treated material and leads to the improvement of their internal boundary interface.

\section{Conclusions}

Using magnetic fields with Zeeman's energy more than three orders smaller than the thermal electron energy at room temperature results in the increase of the optical reflectance of the investigated multilayer structures. Unfortunately, these changes are not stable and the return to the initial state is observed later. The quantitative estimations of the described WMF-induced effects as well as the shift of OR spectra to the blue region require further study. Probably, they are related to the process mentioned in [7]. In this work, the change of the band gap value of the epitaxial layers of $\mathrm{Hg}_{1-\mathrm{x}} \mathrm{Cd}_{\mathrm{x}} \mathrm{Te}$ due to magnetic-field treatment was detected by optical transmittance measurements. 


\section{References}

1. R.B. Morgunov, Spin micromechanics in the physics of plasticity // Phys. Usp. 47, No. 2, p. 125 147 (2004).

2. V.N. Bingi, A.V. Savin, Effects of weak magnetic fields on biological systems: Physical aspects // Phys. Usp. 46, No. 4, p. 259-291 (2003).

3. V.I. Belyavsky, M.N. Levin, Spin effects in defect reactions // Phys. Rev. B, 70, No. 10, p. 104101-1 104101-8 (2004).

4. M.N. Levin, G.V. Semenova, T.P. Sushkova, V.V. Postnikov and B.L. Agapov, Effect of a pulsed magnetic field on the real solid-solution structure in the Sb-As system // Physics of the Solid State, 45, No. 4, p. 639-642 (2003).
5. T.V. Voloshina, M.N. Levin, M.A. Dronov and T.V. Kavetskaya, Effect of a pulsed magnetic field on the luminescence of silver chloride single crystals // Technical Phys. Lett. 32, No. 1, p. 91-93 (2006).

6. R.V. Konakova, S.M. Red'ko, V.V. Milenin, R.A. Red'ko, Effect of weak magnetic fields treatment on photoluminescence of III-V single crystals // Semiconductor Physics, Quantum Electronics and Optoelectronics, 17, No. 1, p. 7579 (2014).

7. A.V. Brodovoi, S.G. Bunchuk and Z.F. Tsybrii, Magnetic-field-induced change in optical transmission spectra of $\mathrm{Hg}_{1-\mathrm{x}} \mathrm{Cd}_{\mathrm{x}} \mathrm{Te}$ epitaxial films // Physics of the Solid State, 53, No. 3, p. 561-563 (2011). 